\title{
Wave-Tilt Characteristics of EM Waves over a Two Layered Earth Model
}

\author{
Ramesh P. SiNGH*, B. U. S. KUMAR**,***, and Tarkeshwar LAL** \\ *Institute of Earth and Planetary Physics, Department of Physics, University of \\ Alberta, Edmonton, Alberta, Canada T6G $2 J 1$ \\ **Department of Geophysics, Banaras Hindu University, Varanasi-221005, India
}

(Received November 29, 1982)

\begin{abstract}
Wave-tilt amplitudes and phase angle variation in the extended frequency range of $10^{4}-10^{7} \mathrm{~Hz}$ have been presented for a two layer earth surface model. The effect of conductivity and dielectric constant variation of two layers on wavetilt amplitude and phase angle have been computed. It is shown to vary with changing thickness of the upper layer. The potentiality of such curves in wavetilt measurement of the subsurface layer has been discussed.
\end{abstract}

\section{Introduction}

In the recent past, the wave-tilt method has emerged as a potential electromagnetic field technique for the determination of near subsurface electrical properties (MALEY 1971; HoEKSTRA et al. 1974; PALACKY and JAGODITS 1975; KING 1976; HoEKSTRA 1978; ARCONE 1978, 1979; THIEL 1979; RALSTON and WAIT 1980; CROSSLEY 1981). The electric and magnetic wave-tilt characteristics over homogeneous and stratified earth models have been studied theoretically by WAIT (1970), Hughes and WAit (1975), LytLe and LAger (1976), Lytle et al. (1976), SinHA (1977), SingH (1980), SingH and LAL (1980, 1981, 1982), and MAHMOUD (1981). Such studies have revealed considerable influence of conductivity and dielectric constant of the subsurface on the amplitude and phase measurements of wave-tilt. Apart from these, the lateral inhomogeneities present in near subsurface and the lateral extent of conductivity of subsurface layer also play an important role (Hughes and WAIT 1975; THIEL et al. 1982). The depth of penetration is governed by the range of frequency used in the experiment and in addition to this attenuation, the angle of incidence and polarization of electromagnetic waves considerably affect the amplitude and phase of the wave-tilt measurements. Recently THIEL and CHANT (1982) observed and reported the influence of iono-spheric reflections on the very low frequency electric wave-tilt measurements.

LYTLE et al. (1976) analysed the wave-tilt characteristics over a half space and n-layered subsurface models and illustrated the amplitude and phase changes

***Present address: Oil and Natural Gas Commission, Nazira, Assam, India. 
in wave-tilt for specific cases of homogeneous, two and three layered subsurface models especially in higher frequency ranges $(1-20 \mathrm{MHz})$. Because of small skin depth in the radio frequency range the stratified subsurface does not play any important role. It is well known that the subsurface penetration capability of low frequency waves increases and the wave-tilt amplitude and phase angle measurements contain information about the stratified subsurface layers. Therefore, in this paper we have extended the frequency range of investigation and have considered the two layer subsurface model. It is shown that the amplitude and phase change of wave-tilt of low and high frequency signals propagating over a given two layer surface behave quite differently. The increasing thickness of the interface adjacent layer undergoes significant amplitude and phase oscillation which are not seen at the lower frequency region. It is probably because the thickness of the interface adjacent layer is too thin in the wavelength scale. The probing and exploration potentiality of computed results have been discussed.

\section{Basic Formulae}

We consider a planer two layered earth with the interface adjacent subsurface layer of thickness $h_{1}$ overlying a semi-infinite earth's surface. Both the layers are assumed to be homogeneous and infinite in the lateral extent. In order to get maximum effect the propagation of plane electromagnetic waves along the earth's surface at grazing incidence is considered. The wave-tilt expressions for the stratified subsurface model have been given by WAIT (1970). The magnitude of wave-tilt for TM and TE polarized waves are written in terms of effective impedance and admittance of a given first subsurface layer as affected by the presence of lower subsurface layers.

$$
W_{\mathrm{e}}=\frac{E_{\mathrm{h}}}{E_{\mathrm{z}}}=\frac{Z_{1}}{\eta_{0}}
$$

and

$$
W_{\mathrm{m}}=\frac{H_{\mathrm{v}}}{H_{\mathrm{h}}}=\frac{1}{Y_{1} \eta_{0}}
$$

where $Z_{1}$ and $Y_{1}$ are effective impedance and admittance of the first layer adjacent to the interface. The $Z_{1}$ and $Y_{1}$ are affected by the physical characteristics and thickness of underlying layers and these are expressible in terms of well known recurrence formulae (WAIT 1970; LytLE et al. 1976). For the sake of ready reference and discussion of results, we express $Z_{1}$ and $Y_{1}$ for the two layer model in terms of standard notations used by WAIT (1970) and LYTLE et al. (1976).

$$
Z_{1}=K_{1} \frac{Z_{1}+K_{1} \operatorname{tanhu}_{1} \mathrm{~h}_{1}}{K_{1}+Z_{2} \operatorname{tanhu}_{1} \mathrm{~h}_{1}}
$$


and

$$
Y_{1}=N_{1} \frac{Y_{2}+N_{1} \operatorname{tanhu}_{1} \mathrm{~h}_{1}}{N_{1}+Y_{2} \operatorname{tanhu}_{1} \mathrm{~h}_{1}}
$$

Using the two layer subsurface model we have computed the $W_{\mathrm{m}}$ and $W_{\mathrm{e}}$ amplitudes and their phase angles in the extended frequency range varying from $10^{4}-10^{7} \mathrm{~Hz}$.

\section{Results and Discussion}

The earth's surface for many practical purposes can be considered as a two layer model. The conductivity of the thin upper surface changes from time to time due to moisture absorption. The penetration of electromagnetic waves over the earth's surface is considerably affected by this surface. The role of the second surface layer comes into being only when the upper surface thickness is smaller than the penetration depth. At lower frequencies the penetration depth for a given conductivity of the layer is much larger as compared to the penetration depth at higher frequency. In order to study this effect we have arbitrarily varied the thickness of the upper surface layer in addition to the conductivity and dielectric constant of the two layers. In the two layer model, basically two combinations of conductivity contrasts are feasible. The one in which the top layer is less conducting than the substratum $\left(\sigma_{1}<\sigma_{2}\right)$ and the other which corresponds to $\sigma_{1}>\sigma_{2}$ i.e. the top layer more conducting than the substratum. We have selected four situations representative of the different possible conductivity combinations such that the conductivity of one of the layers is kept constant while that of the other is varied. The results are presented in Figs. 1 and 2.

Figures 1 and 2 show the variation of amplitude and phase of the wave-tilt frequency for both the modes keeping the conductivity of the second semi-infinite layer constant and varying the interface adjacent layer conductivity. Figure 1 depicts the results for the model $\sigma_{1}<\sigma_{2}$. It is observed that the amplitude of wave-tilt for both TM and TE modes increases as the conductivity contrast of the two layers increases. It is further noticed that the amplitudes of the TE mode are larger than the corresponding amplitudes for the TM mode in the entire frequency range. For smaller conductivity constant $\left(\sigma_{1}=10^{-2}, \sigma_{2}=10^{-1}\right.$ mho $\mathrm{m}^{-1}$ ) the amplitudes are found to increase gradually with the frequency whereas for the larger conductivity contrasts $\left(\sigma_{1}=10^{-3}, 10^{-4}\right.$ and $\left.\sigma_{2}=10^{-1} \mathrm{mho} \mathrm{m}^{-1}\right)$ the amplitudes increase rather smoothly up to $10^{6} \mathrm{~Hz}$. Between $10^{6} \mathrm{~Hz}-10^{7}$ $\mathrm{Hz}$ the amplitude increases sharply, obtaining a maximum value at $10^{7} \mathrm{~Hz}$ and showing an oscillatory character thereafter. The phase of wave-tilt is shown in the lower part of Fig. 1. The phase changes are found to decrease and the decrease is more pronounced with decreasing conductivity of the upper layer. As such the wave-tilt amplitude is found to increase with decreasing phase changes. 


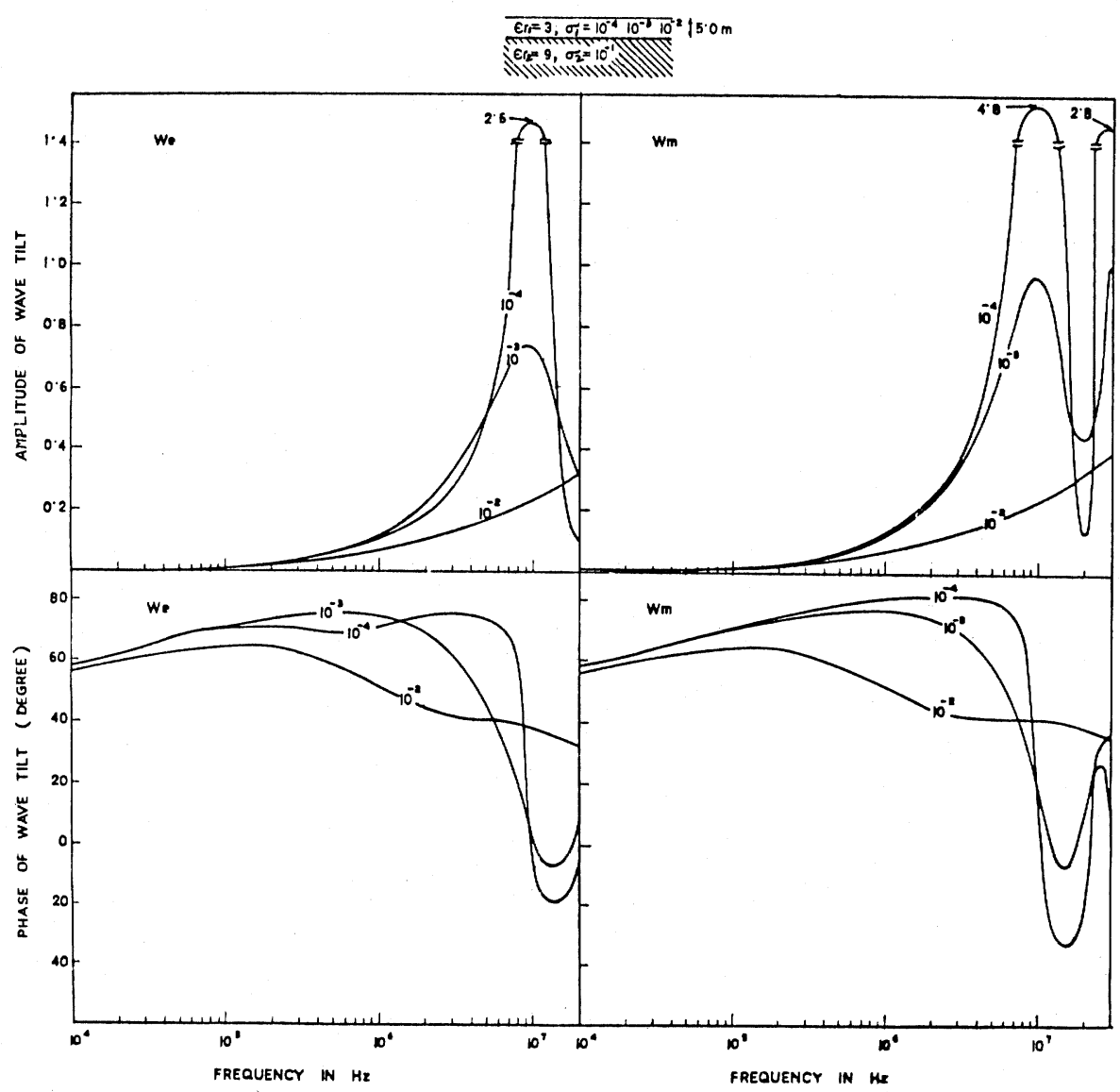

Fig. 1. Variation of amplitude and phase of wave-tilt with frequency when $\sigma_{1}<\sigma_{2}$ keeping $\sigma_{2}$ constant and varying $\sigma_{1}$.

Figure 2 shows the characteristic curves of amplitude and phase of wave-tilt when the top layer is more conductive i.e. $\sigma_{1}>\sigma_{2}$. In this case the wave-tilt amplitude variation is much less pronounced with decreasing conductivity. The $W_{\mathrm{e}}$ and $W_{\mathrm{m}}$ variations depict almost similar variations in the entire frequency range $10^{4}-10^{7} \mathrm{~Hz}$. The phase changes which oscillate with frequency are greatly pronounced in the case $\sigma_{1}>\sigma_{2}$. This happens because the major part of the incident wave propagates through the upper layer and a significant part of the propagating energy is not reflected from the semi-infinite layer characterized by $\sigma_{2}$. In such cases the phase measurements may play an important role in the analysing of the field data. The nature of the variations shown in Fig. 1 and Fig. 2 is capable of deciphering whether $\sigma_{1}>\sigma_{2}$ or $\sigma_{1}<\sigma_{2}$. Further, the thickness 


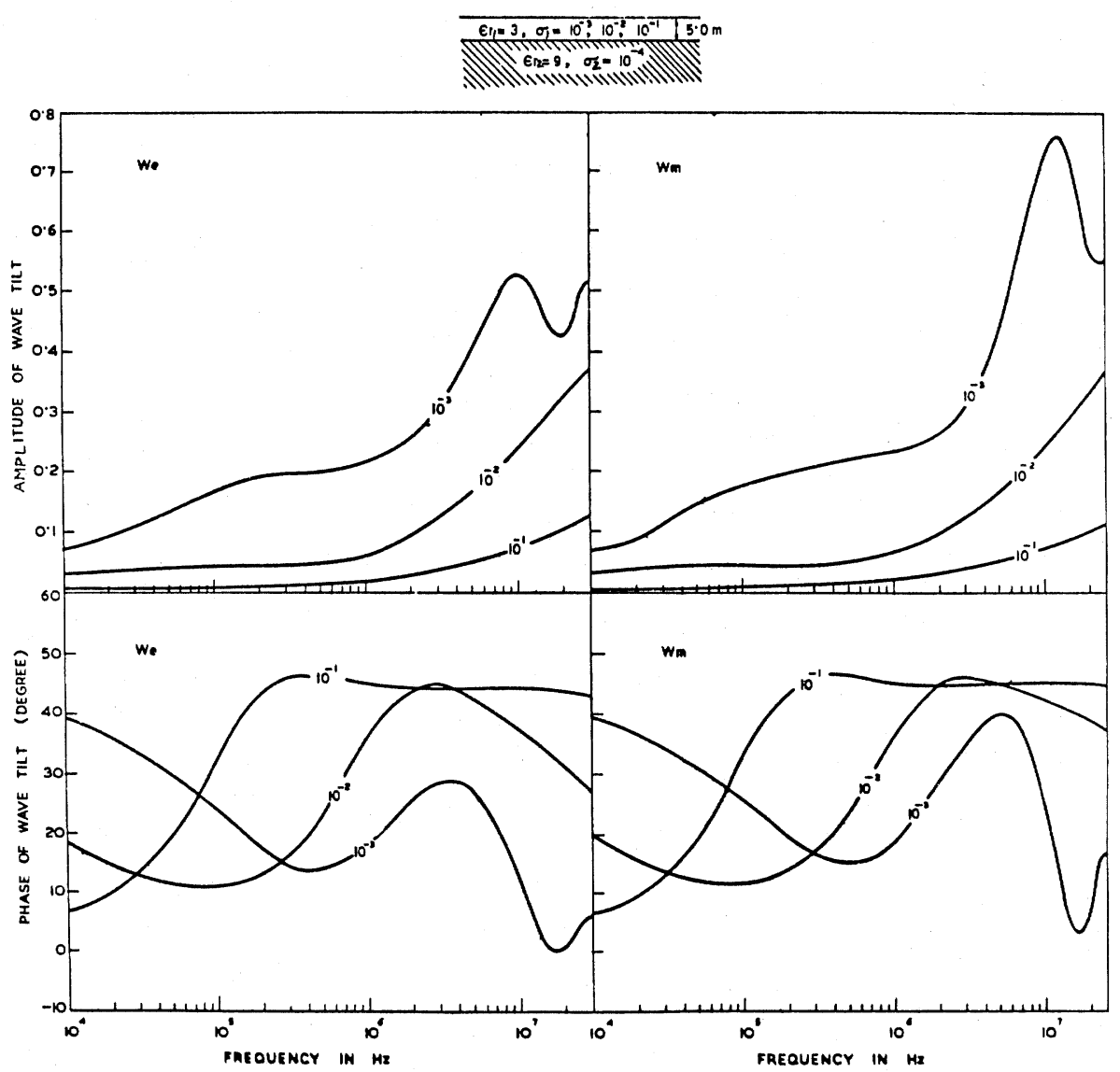

Fig. 2. Variation of amplitude and phase of wave-tilt with frequency when $\sigma_{1}>\sigma_{2}$ keeping $\sigma_{2}$ constant and varying $\sigma_{1}$.

of the upper layer governs the magnitude of wave-tilt amplitude and phase change in the entire frequency range.

We now consider an initial case of $\sigma_{1}<\sigma_{2}$ and the upper layer of constant thickness, say $5 \mathrm{~m}$, which is much smaller than the skin depth corresponding to the chosen conductivity $\sigma_{1}=10^{-3} \mathrm{mho}^{-1}$. The changing percentage of moisture content in the uppermost layer may drastically change the dielectric constant of the layer. Keeping this situation in view, we have studied the effect of varying dielectric constant of the uppermost layer as backed up by a semiinfinite layer of constant conductivity and dielectric constant. The computed wavetilt amplitude and phase change for $W_{\mathrm{e}}$ and $W_{\mathrm{m}}$ are shown in Fig. 3. The amplitude 


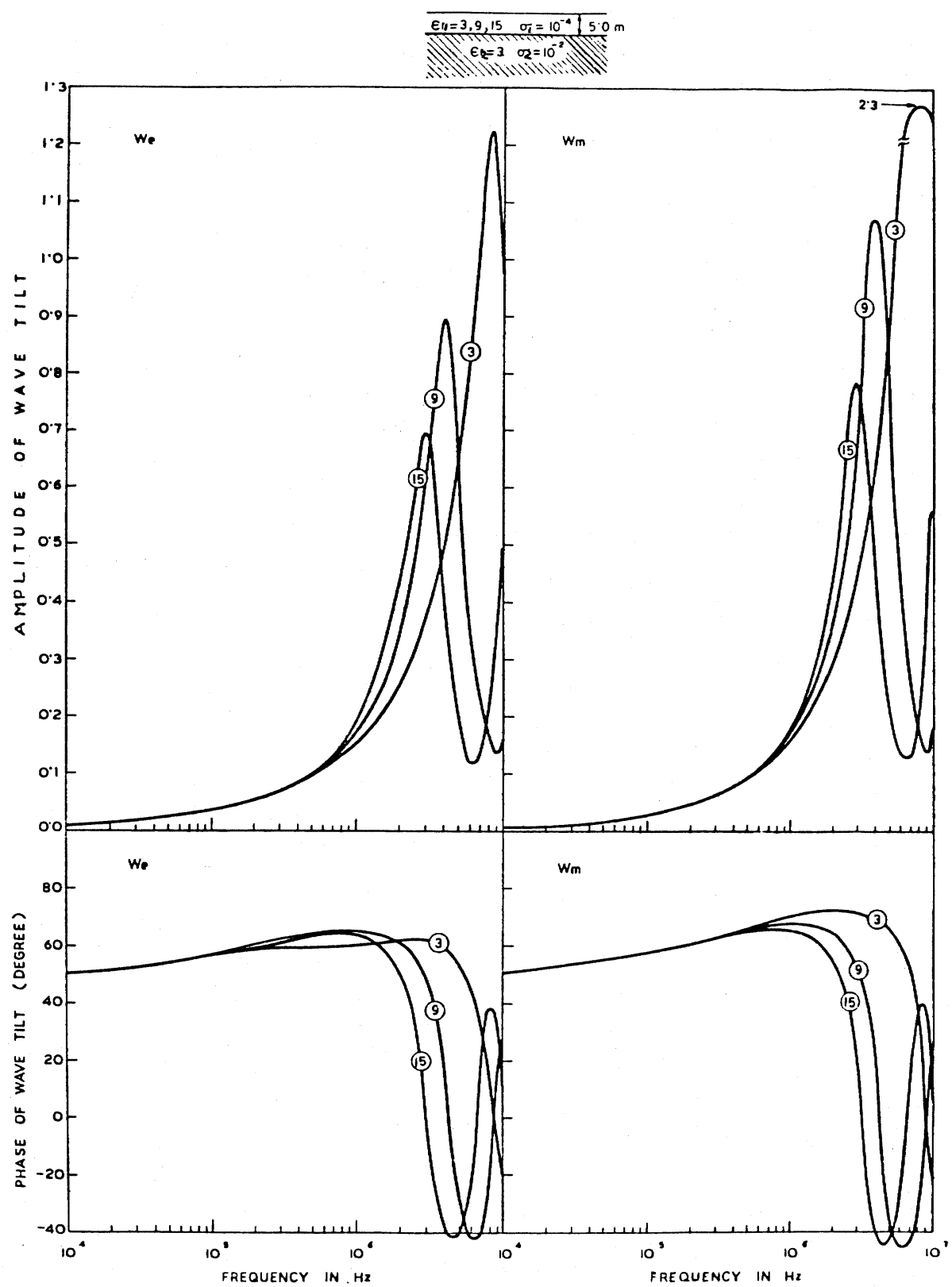

Fig. 3. Variation of amplitude and phase of wave-tilt with frequency with $\epsilon_{\mathrm{r} 1}$ is varied keeping $\varepsilon_{\mathrm{r} 2}$ constant. 
and phase change show almost inverse situations. The characteristic feature of this wave is that the maximum amplitude of wave-tilt decreases with increasing dielectric constant. The maximum amplitude of wave-tilt $W_{\mathrm{e}}$ is seen to appear at different frequencies, namely, 3,4 and $9 \mathrm{MHz}$ for $\varepsilon_{\mathrm{r}}=15,9$ and 3 respectively. The phase changes attain minimum values at different frequencies for different values of $\varepsilon_{\mathrm{r}}$ as shown in Fig. 3. The magnitude of phase is not significantly affected by the changes in the dielectric constant values. Except for changes in the magnitude of $W_{\mathrm{m}}$ and its phase change, the variations are similar to that shown in Fig. 3. The skin depth of the layer changes significantly with the conductivity but it changes rather slowly with the dielectric constant except at high frequency ends. The changing dielectric constant of the uppermost layer may change the effective thickness of the uppermost layer and thus affect the wavetilt response for $W_{\mathrm{e}}$ and $W_{\mathrm{m}}$. The impedance and admittance of the layer also change as depicted by Eqs. (3) and (4).

The effect of varying thickness of the uppermost layer on the wave-tilt amplitude and phase angle has been studied. The computed results have been shown in Fig. 4. The wave-tilt amplitude variation with frequency is seen to increase slightly with increasing thickness. At lower frequencies, the thicknesses used in this computation are much smaller, therefore it does not affect the wavetilt amplitude at lower frequencies. The increasing thickness increases the wavetilt amplitude slightly with increasing thickness. At higher frequencies the penetration depth decreases and becomes comparable with the thickness of the layer. At $5 \mathrm{~m}$ thickness the effect is maximum and for higher thickness the wave-tilt amplitude decreases because the penetration depth at these frequencies is smaller. A similar effect is seen for $W_{\mathrm{m}}$. The phase variation curves show a corresponding variation. At low frequencies the phase change for larger thickness of the uppermost layer is large and decreases with increasing frequencies. At $10^{5} \mathrm{~Hz}$ the phase changes for different layers are quite different and at higher frequencies oscillations appear. The measurement of phase difference between $10^{6}$ and $10^{7} \mathrm{~Hz}$ is capable of providing better resolution.

\section{Concluding Remarks}

The wave-tilt amplitude and phase angle variations are found to be significantly different for various chosen sets of electrical parameters and thicknesses of the uppermost layer. Careful choices of operating frequency range, borehole measurements, conductivity and dielectric constant variation with depth are important parameters in enhancing the wave-tilt capability for subsurface mapping and exploration. The measured wave-tilt amplitude and phase change data, when compared with model computations, are capable of revealing the subsurface conductivity, dielectric constant and depth of the subsurface layers. The effect of dielectric constant variation on wave-tilt amplitude and phase can be analysed with the help of mixture theory to predict the presence of moisture content, mineral content or other subsurface-forming materials. Thus we find that the 


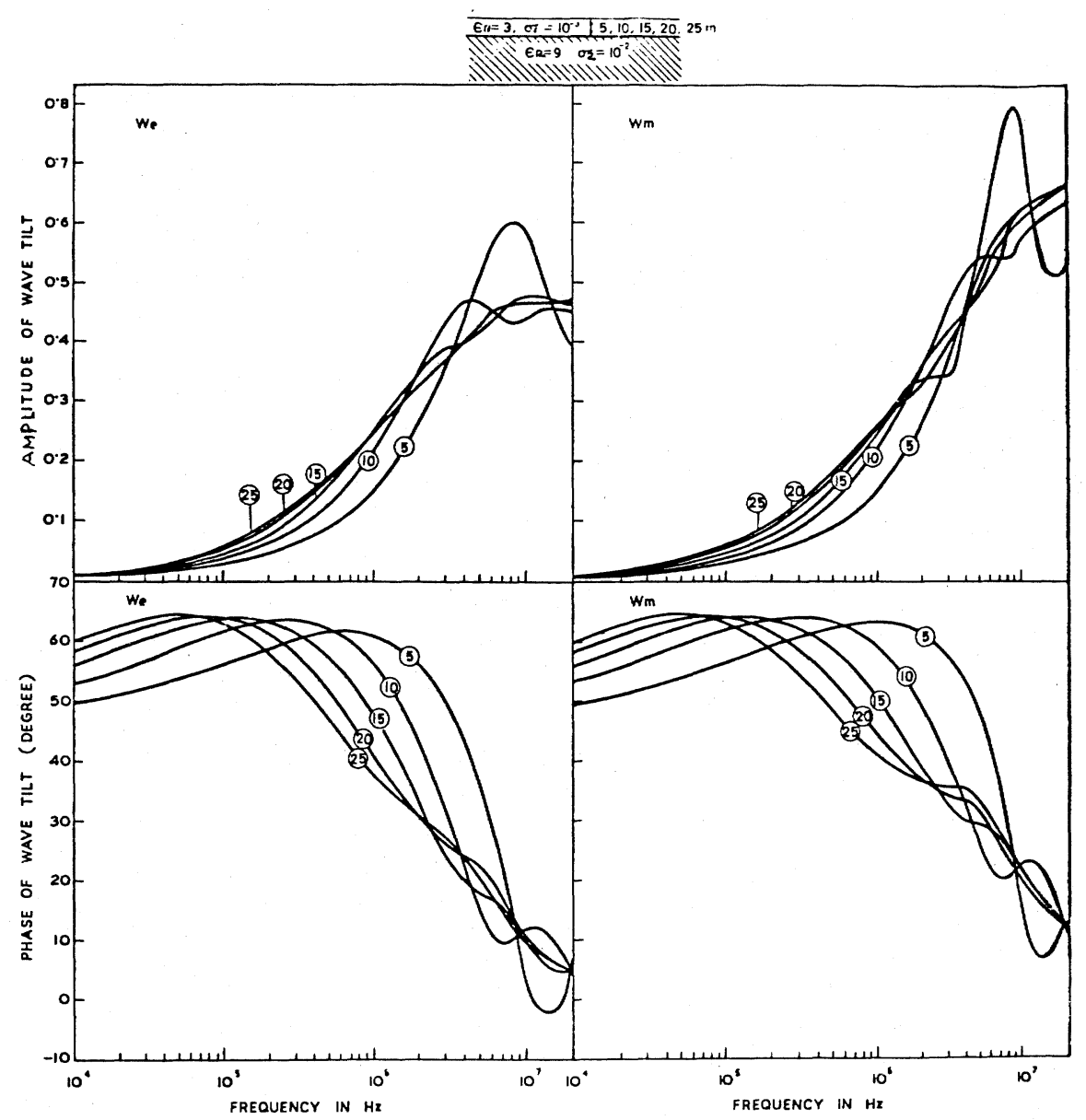

Fig. 4. Variation of amplitude and phase of wave-tilt with frequency when the thickness of the surface layer is varied.

master curves incorporating various types of conductivity and dielectric constant contrast of uppermost subsurface and semi-infinite layers are important in enhancing the exploration capability of the wave-tilt method.

Two of us (RPS, BUSK) are grateful to the Council of Scientific Industrial Research, New Delhi, for financial support. 


\section{REFERENCE}

ARCONE, S. A., Investigation of a VLF airborne resistivity survey conducted in northern Maine, Geophys., 43(7), 1399-1417, 1978.

ARCONE, S. A., Resolution studies in airborne resistivity surveying at VLF, Geophys., 44(5), 937-946, 1979.

Crossley, D. J., The theory of EM surface wave impedance measurements, in Geophysical $A p$ plications of Surface Wave Impedance Measurements, L. S. Collett and O. G. Jensen (eds.), Geological Survey of Canada, paper 81-85, Report V, pp. 35-48, 1981.

HoEkSTRA, P., Electromagnetic methods for mapping shallow permafrost, Geophys., 43(4), 782-787, 1978.

Hoekstra, P., P. V. Sellmann, and A. J. Delaney, Airborne resistivity mapping of permafrost near Fairbanks, Alaska. U. S. Army, Corps of Engineers, Cold Regions Research and Engineering Laboratories, Hanover, N. H., Research Report 324, ADA000694, 1974.

HughES, W. J. and J. R. WAIT, Effective wave-tilt and surface impedance over a laterally inhomogeneous two-layer earth, Radio Sci., 10(11), 1001-1008, 1975.

KING, R. J., Wave-tilt measurements, IEEE Trans. on Antennas and Propagation, AP-24, 115-119, 1976.

LYTLE, R. J. and D. L. LAGER. Theory relating to remote electromagnetic probing of a nonuniformthickness coal seam, Radio Sci., 11(5), 465-475, 1976.

LyTle, R. J., D. L. LAGER, and E. F. LAINE. Subsurface probing by high-frequency measurements of the wave-tilt of electromagnetic surface waves, IEEE Trans. on Geoscience Electronics, GE-14, 244-249, 1976.

MAHMOUD, S. F., Electromagnetic scattering by a layered earth with arbitrarily varying subsurface impedance, Radio Sci., 16(1), 47-53, 1981.

MALEY, S. W., Radio wave methods for measuring the electrical parameters of the earth, in Electromagnetic Probing in Geophysics, edited by J. R. Wait, pp. 77-95, Golem, Boulder, Colorado, 1971.

PALACKY, G. J. and F. L. JAGODITS, Computer data processing and quantitative interpretation of airborne resistivity survey, Geophys., 40(5), 818-830, 1975.

RALSTON, M. A. and J. R. WAIT, Theory of low frequency conductivity probing of roof structures in coal mines, Radio Sci., 15, 1105-1107, 1980.

SinHA, A. K., Influence of altitude and displacement currents on plane-wave EM fields, Geophys., 42, 77-91, 1977.

SINGH, R. P., Studies on electromagnetic response of the subsurface and measurements of dielectric properties of rocks, Ph. D. thesis, Banaras Hindu University, Varanasi, India, 1980.

SINGH, R. P. and T. LAL, Wave-tilt characteristics of electromagnetic waves over a homogeneous earth, IEEE Trans. Geoscience and Remote Sensing, GE-18, 285-287, 1980.

SINGH, R. P. and T. LAL, Wave-tilt characteristics of TE-mode waves. Canadian J. Earth Sci., 18(2), 382-385, 1981.

SINGH, R. P. and T. LAL, Influence of permeability on wave-tilt of EM waves, Canadian J. Earth Sci., 19(6), 1323-1325, 1982.

THIEL, D. V. Relative wave-tilt measurements at VLF, Geoexploration, 17, 285-292, 1979.

THIEL, D. V. and I. J. CHANT, Ionospheric induced very low-frequency field wave-tilt changes, Geophys., 47(1), 60-62, 1982.

THIEL, D. V., K. S. PARK, and R. J. KING, Wave-tilt fluctuations near a vertical discontinuity in a flat ground plane, IEEE Trans. Geoscience and Remote Sensing, GE-20(1), 131-134, 1982.

WAIT, J. R., Electromagnetic Waves in Stratified Media, Pergamon Press, New York, N. Y., 1970. 